\title{
SEESAW MECHANISM AND THE BARYON ASYMMETRY
}

\author{
M. RAIDAL \\ NICPB, Ravala 10, 10143 Tallinn, Estonia
}

\begin{abstract}
I review the present understanding of connection between the non-zero neutrino masses and the baryon asymmetry of the Universe. The state-of-art results are presented for the standard thermal leptogenesis.
\end{abstract}

\section{Smallness of neutrino masses and the seesaw mechanism}

Non-zero neutrino masses and mixing angles provide at the moment the only convincing evidence ${ }^{1}$ of physics beyond the standard model. A paradigm to understand the smallness of neutrino masses, involving some new heavy states which break lepton number, is called the seesaw mechanism ${ }^{2}$. If the heavy particles couple to the Standard Model lepton and Higgs doublets, decoupling of them generates at low scale the dimension five operator

$$
\frac{1}{\Lambda} L L H H
$$

After the electroweak symmetry breaking (1) generates small neutrino masses suppressed by the heavy scale $\Lambda$.

According to the original proposal ${ }^{2}$, which still is by far the most popular and the most studied version of the seesaw mechanism, the heavy states are three superheavy singlet Majorana neutrinos $N_{i}$. These can be identified with the right-handed chiral fields of some grand unification gauge group such as $S O(10)$ and play a fundamental role in the anomaly cancellation. As the singlets do not have the Standard Model gauge couplings, they do not spoil the nice features of the Standard Model or its supersymmetric extension, such as the gauge coupling unification. The relevant terms for the light neutrino masses in the Lagrangian are the neutrino Yukawa couplings $Y_{\nu}$ and Majorana masses $m_{N}$,

$$
L=\bar{L}_{L i} Y_{\nu}^{i j} N_{R j} H+\frac{1}{2} \bar{N}_{R i}^{c} m_{N}^{i j} N_{R j}+\text { h.c. }
$$


Integrating out the heavy neutrinos one obtains the seesaw relation for light neutrino masses

$$
m_{\nu}=-Y_{\nu}^{T} m_{N}^{-1} Y_{\nu} v^{2}
$$

where $v=174 \mathrm{GeV}$. According to that, the relation between light neutrino masses and mixing in $m_{\nu}$, and the structure of the heavy neutrino Yukawa couplings and mass matrix is rather complicated, and obtaining the observed neutrino mixing pattern requires complicated flavour model building ${ }^{3}$. As the deviation of the light neutrino mixing from bimaximal seems to be parametrized by the quark mixing matrix, this quark-lepton complementarity ${ }^{4}$ may indicate some unification effect for the fermion Yukawa couplings. There are 9 physical degrees of freedom in the left-hand side of (3) while the right-hand side contains 18 of them. The missing 9 physical degrees of freedom at low energies can be parametrized by an orthogonal parameter matrix $R^{5}$ or by an Hermitian parameter matrix $H^{6}$. The latter one allows to relate the missing degrees of freedom to different observables in the supersymmetric models with universal boundary condition for the soft supersymmetry breaking terms ${ }^{7}$. Those include the renormalization induced lepton flavour violating processes ${ }^{8}$ and electric dipole moments ${ }^{9}$. The connection between low- and high-energy observables in the singlet seesaw models has been reviewed by S. Davidson in this conference ${ }^{10}$.

The second proposal for generating light neutrino masses is to couple the Standard Model doublets to the $S U(2)_{L}$ triplet Higgs boson with non-zero hypercharge ${ }^{11}$. The relevant interaction terms are given by

$$
L=\frac{1}{\sqrt{2}}\left(Y_{T}^{i j} \bar{L}_{i}^{c} i \tau_{2} \mathbf{T} L_{j}+\lambda H^{T} \mathbf{T}^{*} i \tau_{2} H+\text { h.c. }\right)+M_{T} \operatorname{Tr}\left[\mathbf{T} \mathbf{T}^{\dagger}\right],
$$

where $\mathbf{T}=\tau \cdot T$, the triplet $T$ is in the $S U(2)_{L} \times U(1)_{Y}$ representation $T \sim(3,1)$, and the $\tau_{i}$ are the three Pauli matrices. Notice that the Yukawa couplings $Y_{T}$ and the Higgs self coupling $\lambda$ together break lepton number explicitly. In this case the neutrino masses are suppressed by the heavy triplet mass $M_{T}$ via the triplet seesaw mechanism ${ }^{12}$

$$
m_{\nu}^{i j}=Y_{T}^{i j} \lambda \frac{v^{2}}{M_{T}}
$$

The triplet neutrino mass mechanism is very appealing one from the low energy neutrino phenomenology point of view because it requires introduction of the minimal number of new degrees of freedom and because the neutrino masses are directly proportional (up to the renormalization 
effects) to the triplet Higgs Yukawa couplings. In the triplet seesaw mechanism the low energy neutrino mass measurements determine directly, up to the overall scale, the structure of Majorana type Yukawa couplings in the fundamental Lagrangian. Indeed, comparison of (5) with (3) shows that the flavour structure of (5) is trivial. Therefore the explanation to the almost bimaximal light neutrino mixing is free of fine tunings and unnatural cancellations between numerical parameters. This simplicity also implies that the flavour violating processes in supersymmetric models are related to each other ${ }^{13}$. The scale of $M_{T}$ can vary from almost unification scale to as low as $1 \mathrm{TeV}^{14}$. In the latter case Higgs triplet can be discovered in the future collider experiments ${ }^{15}$.

The third neutrino mass mechanism with triplet fermions 16 is considered to be somewhat exotic and has not gained much attention in the neutrino model building industry. We do not discuss this possibility in this talk any further.

\section{The seesaw mechanism and leptogenesis}

Another observable related to the physics of neutrino masses is the baryon asymmetry of the Universe. The observed ratio of baryon density to entrophy density ${ }^{17}$,

$$
\frac{n_{B}}{s}=(8.7 \pm 0.4) \times 10^{-11},
$$

requires the existence of physics beyond the Standard Model. To generate (6), three famous Sakharov's conditions must be satisfied ${ }^{18}$. Firstly, baryon number must be violated. Secondly, C and CP must be violated. Thirdly, the process must take place in out-of-equilibrium situation. In principle, those conditions can be satisfied also in the Standard Model since at non-perturbative level both $B$ and $L$ are separately violated ${ }^{19}$. However, baryogenesis in the electroweak phase transition has been extensively studied and found not to able to generate (6) because it requires very light Higgs boson mass $M_{H}<40 \mathrm{GeV}$. Therefore, currently the most widely accepted concept for generating (6) is leptogenesis.

According to the paradigm of leptogenesis ${ }^{20}$, non-zero lepton asymmetry is generated first in out-of-equilibrium decays of some heavy states in the early universe. Thus the interactions of those heavy particles must violate lepton number and $\mathrm{CP}$, and out-of-equilibrium condition is provided by the expansion of the Universe, $\Gamma<H$, where $H$ is the Hubble parameter. Thereafter the lepton asymmetry is reprocessed to the $B-L$ asymmetry 
by the sphaleron processes ${ }^{21}$, generating (6). Baryogenesis via leptogenesis is the only idea which is also supported by the experimental data, namely by the non-vanishing neutrino masses and mixing. If we require the seesaw mechanism to induce simultaneously both the neutrino masses and the baryon asymmetry of the Universe, one can constrain leptogenesis from the experimental neutrino data. From that point of view different realizations of the seesaw mechanism discussed in the previous Section have quite different features. In this talk I consider only thermal leptogenesis, i.e., the case when all the particle species are created by thermal plasma during and after reheating of the Universe. All the following discussion applies only to that case.

In the case of the singlet neutrino seesaw mechanism ${ }^{2}$ leptogenesis ${ }^{20}$ is a direct, almost un-avoidable, consequence of seesaw rather that a separate mechanism. Because present neutrino data requires the existence of at least two heavy singlet neutrinos, and because in the case of Majorana particles physical CP phases exist already in the case of two generation, even the most minimal singlet seesaw model implies viable leptogenesis ${ }^{22}$. If the heavy and light neutrinos are hierarchical in mass so that leptogenesis comes from the decays of the lightest singlet $N_{1}$ only, there exist an upper bound on the $\mathrm{CP}$ asymmetry from its decay ${ }^{23}$

$$
\epsilon_{N_{1}} \leq \frac{3}{16 \pi} \frac{m_{N_{1}} m_{3}}{v^{2}}
$$

where $m_{3}$ is the heaviest light neutrino mass. In more general case there is an upper bound on the the light neutrino mass scale from leptogenesis ${ }^{24}$ which does not allow highly degenerate neutrino masses. This, together with (6) and with the state-of-art estimates of the thermal washout effects ${ }^{25}$, leads to the lower bound on the leptogenesis scale, which turns out to be $m_{N_{1}}>2 \times 10^{9} \mathrm{GeV}$. However, if the singlet neutrinos are partially degenerate in mass, the CP asymmetry is resonantly enhanced ${ }^{26,27}$ and leptogenesis scale as low as $O(1) \mathrm{TeV}$ could be viable. In principle the bound (7) can also be violated if the heavy neutrinos are not degenerate ${ }^{28}$. First neutrino model of that sort has been proposed in Ref. ${ }^{29}$, and such models are quite different from the generic ones. All-together, leptogenesis is a very natural consequence of the singlet seesaw mechanism.

On the other hand, the triplet seesaw mechanism, which in its minimal form contains just one triplet Higgs, does not provide leptogenesis in the Standard Model because of the lack of interfering amplitudes. The minimal triplet leptogenesis model must contain two triplets ${ }^{30}$ which doubles the neutrino degrees of freedom, and the nicest phenomenological argument of 
simplicity in favour of this scenario is lost. However, in the minimal supersymmetric version of the triplet seesaw model leptogenesis is possible ${ }^{31}$. In the supersymmetric triplet seesaw model the anomaly cancellation requires introduction of two triplets with opposite $U(1)$ quantum numbers, $T \sim(3,1)$ and $\bar{T} \sim(3,-1)$. According to the superpotential

$$
W=\frac{1}{\sqrt{2}}\left(Y_{T}^{i j} L_{i} T L_{j}+\lambda_{1} H_{1} T H_{1}+\lambda_{2} H_{2} \bar{T} H_{2}\right)+M_{T} T \bar{T},
$$

$T$ and $\bar{T}$ have equal masses but only one of them couples to the lepton doublet, thus giving (5) with $\lambda=\lambda_{2}, v=v_{2}$. When supersymmetry breaking terms are included, $T$ and $\bar{T}$ degeneracy is split by the soft terms, and resonant leptogenesis occurs ${ }^{31}$ via the mechanism called "soft leptogenesis" 32. While the scale of triplet seesaw is rather arbitrary, the scale of triplet leptogenesis is very much constrained due to the strong dependence on the size of the soft supersymmetry breaking $B$ terms. In the following discussion we concentrate only on the singlet leptogenesis as the most interesting one.

\section{Standard thermal leptogenesis}

By the standard leptogenesis we mean the evolution of lepton asymmetry in the thermal plasma during and after reheating of the Universe. The heavy neutrinos are assumed to be hierarchical in mass so that leptogenesis occurs in the decays of the lightest heavy neutrinos $N_{1}$ only. Despite of the fact that the neutrino sector of the minimal seesaw model contains 18 free parameters, this scenario depends on only very few combinations of them. We parametrize the generated $B-L$ asymmetry via

$$
Y_{B-L}=-\epsilon_{N_{1}} \eta Y_{N_{1}}^{\mathrm{eq}}\left(T \gg m_{N_{1}}\right)
$$

where $\epsilon_{N_{1}}$ is the CP-asymmetry in $N_{1}$ decays at zero temperature given by 27

$$
\epsilon_{N_{1}}(T=0)=\frac{1}{8 \pi} \sum_{j \neq 1} \frac{\operatorname{Im}\left[\left(Y^{\dagger} Y\right)_{j 1}^{2}\right]}{\left[Y^{\dagger} Y\right]_{11}} f\left(\frac{m_{N_{j}}^{2}}{m_{N_{1}}^{2}}\right)
$$

with

$$
f(x)=\sqrt{x}\left[\frac{x-2}{x-1}-(1+x) \ln \left(\frac{1+x}{x}\right)\right] \stackrel{x \gg 1}{\longrightarrow}-\frac{3}{2 \sqrt{x}},
$$

and $Y_{N_{1}}^{\mathrm{eq}}\left(T \gg m_{N_{1}}\right)=135 \zeta(3) /\left(4 \pi^{4} g_{*}\right)$ is the neutrino equilibrium density

at high temperature. Here $g_{*}$ counts the effective number of spin-degrees 
of freedom in thermal equilibrium $\left(g_{*}=106.75\right.$ in the SM with no righthanded neutrinos). If the light (and also heavy) neutrino masses are taken to be hierarchical, the lower bound (7) on the $\mathrm{CP}$ asymmetry holds. In the following we assume that this is the case. Eq.(9) can be regarded as the definition of the leptogenesis efficiency parameter $\eta$, which contains all the finite temperature effects and dependences on the initial conditions for heavy neutrino abundances. For hierarchical neutrino masses, and for particular initial condition, the efficiency $\eta$ depends on only two parameters. Those are $m_{N_{1}}$ and the effective neutrino mass

$$
\tilde{m}_{1}=\frac{\left(Y_{\nu} Y_{\nu}^{\dagger}\right)_{11}}{m_{N_{1}}} v^{2}
$$

which is the measure on $N_{1}$ interaction strength with thermal plasma.

The obtained $B-L$ asymmetry is converted to the baryon asymmetry via sphaleron processes

$$
\frac{n_{B}}{s}=\frac{24+4 n_{H}}{66+13 n_{H}} \frac{n_{B-L}}{s},
$$

where $n_{H}$ is the number of Higgs doublets. For the SM we find numerically

$$
\frac{n_{B}}{s}=-1.38 \times 10^{-3} \epsilon_{N_{1}} \eta \text {. }
$$

Although the standard thermal leptogenesis scenario has been studied extensively ${ }^{33}$, several important aspects of this scenario have been worked out in detail quite recently. Most important of them is adding finite temperature corrections to the decay and scattering amplitudes and to the $\mathrm{CP}$ asymmetry ${ }^{25}$. It has been found that to a good approximation the dominant effects are included if one (i) uses thermal masses of particles in the processes involved instead of zero temperature masses; (ii) renormalizes all the couplings at the first Matsubara mode,

$$
E_{r}=2 \pi T,
$$

using the zero-temperature renormalization group equations; (iii) uses finite temperature Feynman rules for calculation of the $\mathrm{CP}$ asymmetries. The important consequence of this is that at high temperatures the Higgs boson mass exceeds the sum of the singlet neutrino and lepton mass, and instead of $N$ decay the two-body decay process is $H \rightarrow N L$. The second important refinement of the calculation is inclusion of the gauge scatterings in 25 (which in particular limit agree with the similar attempt in ${ }^{34}$ ). Thus the 

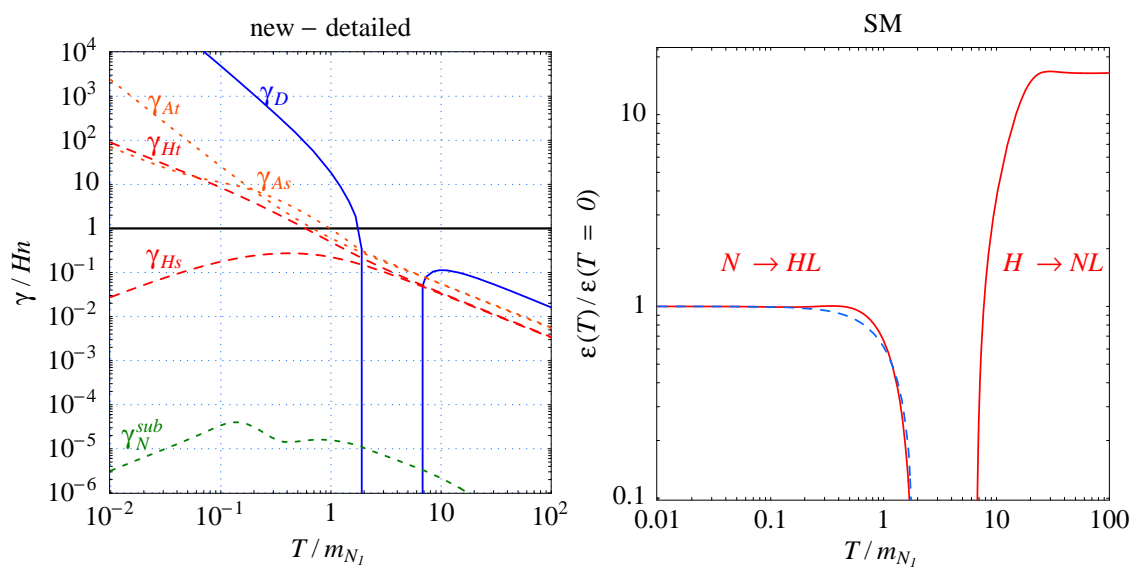

Figure 1. Evolution of the scattering densities (first plot) and the $\mathrm{CP}$ asymmetries (second plot) with temperature.

processes contributing to the thermal leptogenesis are

$$
\begin{array}{ll}
\Delta L=1: & D=\left[N_{1} \leftrightarrow L H\right], \quad S_{s}=H_{s}+A_{s}, \quad S_{t}=H_{t}+A_{t}, \\
\Delta L=2: & N_{s}=[L H \leftrightarrow \bar{L} \bar{H}], \quad N_{t}=[L L \leftrightarrow \bar{H} \bar{H}],
\end{array}
$$

where

$$
\begin{aligned}
& H_{s}=\left[L N_{1} \leftrightarrow Q_{3} U_{3}\right], 2 H_{t}=\left[N_{1} \bar{U}_{3} \leftrightarrow Q_{3} \bar{L}\right]+\left[N_{1} \bar{Q}_{3} \leftrightarrow U_{3} \bar{L}\right], \\
& A_{s}=\left[L N_{1} \leftrightarrow \bar{H} A\right], \quad 2 A_{t}=\left[N_{1} H \leftrightarrow A \bar{L}\right]+\left[N_{1} A \leftrightarrow \bar{H} \bar{L}\right] .
\end{aligned}
$$

We separated $\Delta L=1$ scatterings $S_{s, t}$ into top $\left(H_{s, t}\right)$ and gauge contributions $\left(A_{s, t}\right)$. The evolution of scattering densities $\gamma$ for the particular processes with temperature has been shown in Fig. 1 for $m_{N_{1}}=10^{10}$ $\mathrm{GeV}$ and $\tilde{m}_{1}=0.06 \mathrm{eV}$. It follows that there is a temperature range in which $H$ and $L$ thermal masses are such that all the two-body decays are kinematically forbidden. Notice that in order to avoid double counting of the two-body decays, after subtracting the resonances from the scattering $L H \leftrightarrow L H$ the corresponding $\gamma_{N}^{s u b}$ is completely negligible compared to the other processes. In the same figure we also plot the dependence of the $\mathrm{CP}$ asymmetries in $N \rightarrow H L$ and in $H \rightarrow N L$ decays. The solid line shows the result of full finite temperature calculation in ${ }^{25}$ while the dashed line is obtained by approximating $N_{1}$ to be at rest in thermal plasma. Those results agree with each other with good accuracy. The results including thermal corrections plotted in Fig. 1 differ both qualitatively and quantitatively from the zero temperature calculations and change the predictions 

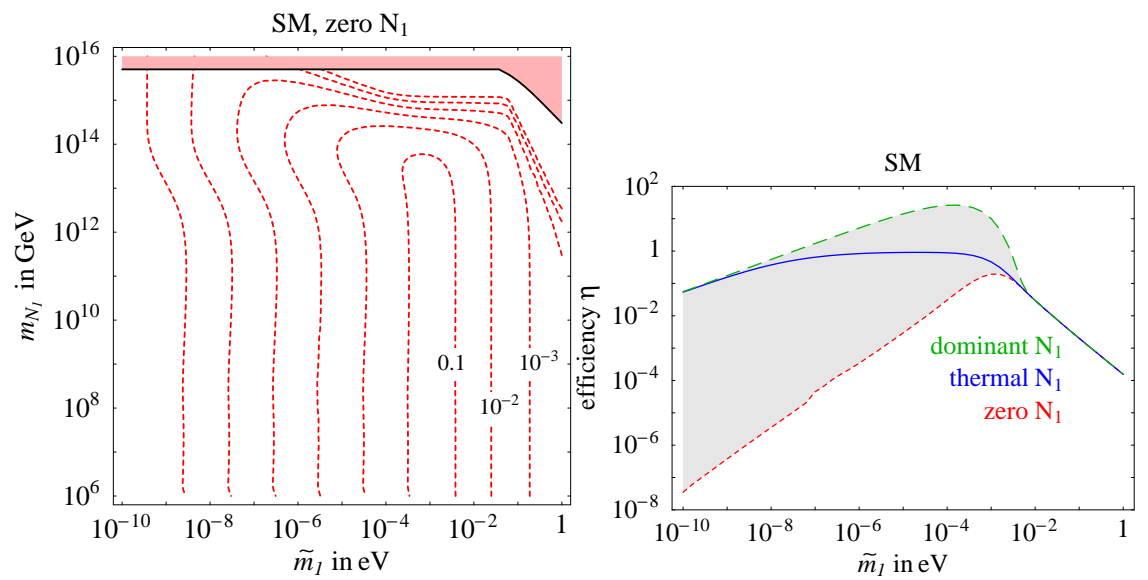

Figure 2. Isocurves of leptogenesis efficiency $\eta$. The first plot is for vanishing initial $N_{1}$ abundance, the second plot is for fixed $m_{N_{1}}=10^{10} \mathrm{GeV}$.

for the generated $B-L$ asymmetry.

Solving the full set of Boltzmann equations for the evolution of the $B-L$ asymmetry with temperature, and parameterizing the results via Eq.(9), our results for the leptogenesis efficiency $\eta$ are shown in Fig. 2. In the first plot we present the isocurves of $\eta=10^{i}, i=-1,-2,-3, \ldots$ in the $\left(\tilde{m}_{1}, m_{N_{1}}\right)$ plane, assuming vanishing initial abundance for the singlet neutrinos $Y_{N_{1}}(T=\infty)=0$. The maximum efficiency is found around $\tilde{m}_{1} \sim$ $10^{-3} \mathrm{eV}$. For smaller values of $\tilde{m}_{1}$ the Yukawa interactions of $N_{1}$ are too weak for $N_{1}$ to reach thermal abundance, which leads to the suppression of the $B-L$ asymmetry. For larger values of $\tilde{m}_{1}$ the Yukawa interaction becomes too strong so that the $N_{1}$ decays occur not sufficiently in out-ofequilibrium, which again leads to the suppression of the $B-L$ asymmetry. Our result can be summarized with a simple analytical fit ${ }^{25}$

$$
\frac{1}{\eta} \approx \frac{3.3 \times 10^{-3} \mathrm{eV}}{\tilde{m}_{1}}+\left(\frac{\tilde{m}_{1}}{0.55 \times 10^{-3} \mathrm{eV}}\right)^{1.16}
$$

valid for $m_{N_{1}} \ll 10^{14} \mathrm{GeV}$. This enables the reader to study leptogenesis in neutrino mass models without setting up and solving the complicated Boltzmann equations.

The dependence of the efficiency on the initial conditions for $N_{1}$ is demonstrated on the second plot in Fig. 2 where we assume $m_{N_{1}}=10^{10}$ $\mathrm{GeV}$ and study three different cases, zero initial abundance for $N_{1}$, thermal initial abundance for $N_{1}$ and when $N_{1}$ dominates the Universe. For 

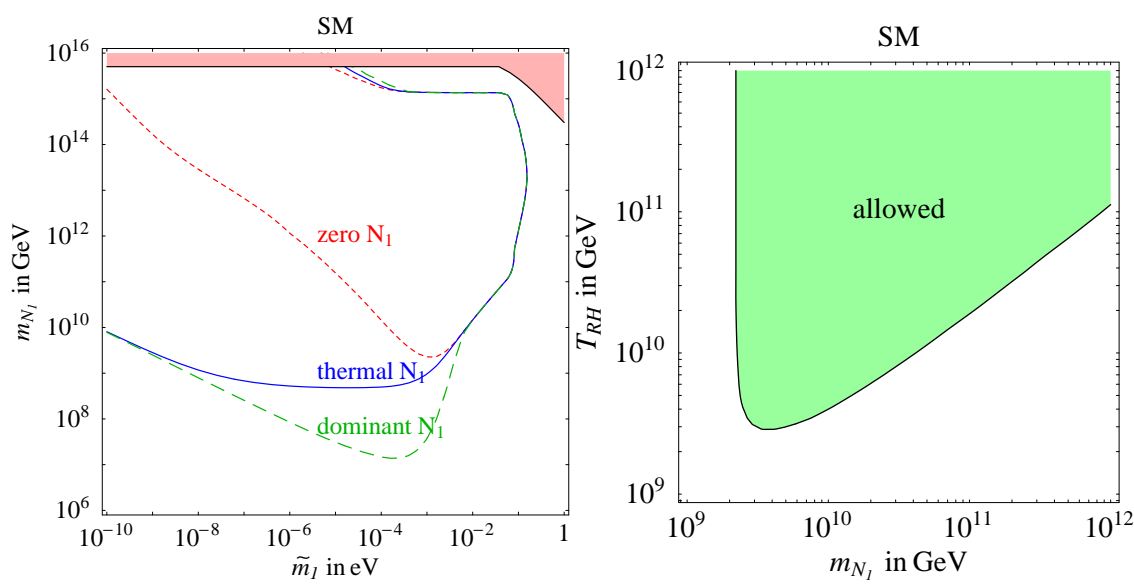

Figure 3. Regions in $\left(\tilde{m}_{1}, m_{N_{1}}\right)$ and $\left(m_{N_{1}}, T_{R H}\right)$ planes allowed by successful leptogenesis.

small values of $\tilde{m}_{1}$ the results depend on the initial conditions. However, if $\tilde{m}_{1}=\sqrt{\Delta m_{\text {sol }}^{2}}$ or $\tilde{m}_{1}=\sqrt{\Delta m_{\text {atm }}^{2}}$, the prediction of thermal leptogenesis is practically independent of any pre-existing initial condition. This makes the thermal leptogenesis predictions so robust.

In order to study in which parameter space the thermal leptogenesis is capable to yield the observed baryon asymmetry of the Universe, one has to combine the results for the efficiency $\eta$ with the maximal value of the $\mathrm{CP}$ asymmetry (corrections to Eq.(7) from non-zero $m_{1}$ have to be taken into account). This has been done in Fig. 3. In the first plot in this figure we present the contours for successful leptogenesis in the $\left(\tilde{m}_{1}, m_{N_{1}}\right)$ plane for the three initial conditions for $N_{1}$ as before. There exist lower bounds on the $N_{1}$ mass from the requirement of successful leptogenesis which depend on the initial conditions. For the vanishing initial $N_{1}$ abundance, which is probably the most appropriate assumption for singlets, the bound is ${ }^{25}$

$$
m_{N_{1}}>2 \times 10^{9} \mathrm{GeV} .
$$

To derive the results presented so far we have assumed that the reheating temperature of the Universe exceeds the singlet neutrino mass, $T_{R H} \gg m_{N_{1}}$. This may not be the case in all the scenarios, especially in supersymmetric ones because supersymmetry sets an upper bound on the reheating temperature of the Universe from the overproduction of gravitinos ${ }^{35}$. Therefore one has to study how our predictions depend on the reheating of the Universe. We have set up and solved Boltzmann equations 
which include $T_{R H}$ as a free parameter in Ref. ${ }^{25}$. The result is presented on the second plot in Fig. 3 where we show the region in the $\left(m_{N_{1}}, T_{R H}\right)$ plane allowed by thermal leptogenesis. We have taken $\tilde{m}_{1}=10^{-3} \mathrm{eV}$ and assumed vanishing initial abundance for $N_{1}$. The results show that $T_{R H}$ can be as low as $m_{N_{1}}$ without considerable loss of the efficiency. However, if $T_{R H}<m_{N_{1}}$ successful leptogenesis requires large values of $m_{N_{1}}$, considerably larger than the minimally allowed one.

\section{Conclusions}

Baryogenesis via leptogenesis, the idea of generating the observed baryon asymmetry of the Universe in out-of-equilibrium decays of heavy particles which violate lepton number, is supported by the light neutrino data. Remarkably, the standard thermal leptogenesis works in particularly robust way in the neutrino parameter space suggested by the seesaw mechanism. Currently it is not possible to make exact predictions between the low energy neutrino measurements and the generated baryon asymmetry because of too many free parameter in the seesaw model. Nevertheless the experimental and theoretical success in understanding the seesaw meshanism during its first 25 years has been impressive. Hopefully in next 25 years, if next generation experiments will find new lepton flavour violating observables, leptogenesis can be experimantally established as the mechanism of baryogenesis.

\section{Acknowledgments}

I would like to thank all my collaborators with whom I have studied the issues presented in this talk over several past years. This work has been supported by the ESF Grants 5135 and 5935, by the EC MC contract MERG-CT-2003-503626, and by the Ministry of Education and Research of the Republic of Estonia.

\section{References}

1. M. H. Ahn et al. [K2K Collab.], Phys. Rev. Lett. 90, 041801 (2003) [arXiv:hep-ex/0212007]; K. Eguchi et al. [KamLAND Collab.], Phys. Rev. Lett. 90, 021802 (2003) [arXiv:hep-ex/0212021]; S. N. Ahmed et al. [SNO Collab.], arXiv:nucl-ex/0309004; M. Ishitsuka [Super-Kamiokande Collaboration], arXiv:hep-ex/0406076.

2. P. Minkowski, Phys. Lett. B 67 (1977) 421; M. Gell-Mann, P. Ramond and R. Slansky, Proceedings of the Supergravity Stony Brook Workshop, New 
York, 1979, eds. P. Van Nieuwenhuizen and D. Freedman (North-Holland, Amsterdam); T. Yanagida, Proceedings of the Workshop on Unified Theories and Baryon Number in the Universe, Tsukuba, Japan 1979 (eds. A. Sawada and A. Sugamoto, KEK Report No. 79-18, Tsukuba); S.L. Glashow, in Quarks and Leptons, Cargèse, eds. M. Lévy et al., (Plenum, 1980, New-York), p. 707; T. Yanagida, in Proceedings of the Workshop on the Unified Theory and the Baryon Number in the Universe, edited by O. Sawada and A. Sugamoto (KEK Report No. 79-18, Tsukuba, 1979), p. 95; R.N. Mohapatra and G. Senjanović, Phys. Rev. Lett. 44, (1980) 912.

3. For reviews see, G. Altarelli and F. Feruglio, Phys. Rept. 320, 295 (1999); G. Altarelli and F. Feruglio, arXiv:hep-ph/0306265; M. C. Gonzalez-Garcia and Y. Nir, Rev. Mod. Phys. 75, 345 (2003) [arXiv:hep-ph/0202058]; A. Y. Smirnov, arXiv:hep-ph/0402264; F. Feruglio, arXiv:hep-ph/0410131.

4. M. Raidal, Phys. Rev. Lett. 93 (2004) 161801 [arXiv:hep-ph/0404046]; H. Minakata and A. Y. Smirnov, arXiv:hep-ph/0405088; P. H. Frampton and R. N. Mohapatra, arXiv:hep-ph/0407139.

5. J. A. Casas and A. Ibarra, Nucl. Phys. B 618 (2001) 171 [arXiv:hep$\mathrm{ph} / 0103065]$.

6. S. Davidson and A. Ibarra, JHEP 0109 (2001) 013 [arXiv:hep-ph/0104076]; J. R. Ellis, J. Hisano, M. Raidal and Y. Shimizu, Phys. Rev. D 66 (2002) 115013 [arXiv:hep-ph/0206110].

7. F. Borzumati and A. Masiero, Phys. Rev. Lett. 57 (1986) 961; L. J. Hall, V. A. Kostelecky and S. Raby, Nucl. Phys. B 267 (1986) 415.

8. J. Hisano, T. Moroi, K. Tobe, M. Yamaguchi and T. Yanagida, Phys. Lett. B 357 (1995) 579 [arXiv:hep-ph/9501407]; J. Hisano, T. Moroi, K. Tobe and M. Yamaguchi, Phys. Rev. D 53 (1996) 2442 [arXiv:hep-ph/9510309].

9. J. R. Ellis, J. Hisano, S. Lola and M. Raidal, Nucl. Phys. B 621 (2002) 208 [arXiv:hep-ph/0109125]; J. R. Ellis, J. Hisano, M. Raidal and Y. Shimizu, Phys. Lett. B 528 (2002) 86 [arXiv:hep-ph/0111324]; Y. Farzan and M. E. Peskin, arXiv:hep-ph/0405214.

10. S. Davidson, arXiv:hep-ph/0409339, these proceedings.

11. J. Schechter and J. W. F. Valle, Phys. Rev. D22 (1980) 2227; T. P. Cheng and L. F. Li, Phys. Rev. D22 (1980) 2860.

12. M. Magg and C. Wetterich, Phys. Lett. B94 (1980) 61; C. Wetterich, Nucl. Phys. B187 (1981) 343; G. Lazarides, Q. Shafi and C. Wetterich, Nucl Phys. B181 (1981) 287; R.N. Mohapatra and G. Senjanović, Phys. Rev. D23 (1981) 165.

13. A. Rossi, Phys. Rev. D 66 (2002) 075003 [arXiv:hep-ph/0207006].

14. E. Ma, M. Raidal and U. Sarkar, Phys. Rev. Lett. 85 (2000) 3769 [arXiv:hep$\mathrm{ph} / 0006046]$.

15. K. Huitu, J. Maalampi, A. Pietila and M. Raidal, Nucl. Phys. B 487 (1997) 27 [arXiv:hep-ph/9606311]; F. Cuypers and M. Raidal, Nucl. Phys. B 501 (1997) 3 [arXiv:hep-ph/9704224].

16. E. Ma, Phys. Rev. Lett. 81 (1998) 1171 [arXiv:hep-ph/9805219].

17. H. V. Peiris et al., Astrophys. J. Suppl. 148 (2003) 213 [arXiv:astro$\mathrm{ph} / 0302225]$. 
18. A. D. Sakharov, Pisma Zh. Eksp. Teor. Fiz. 5 (1967) 32 [JETP Lett. 5 (1967 SOPUA,34,392-393.1991 UFNAA,161,61-64.1991) 24].

19. G. 't Hooft, Phys. Rev. Lett. 37 (1976) 8.

20. M. Fukugita and T. Yanagida, Phys. Lett. B 174 (1986) 45.

21. V. A. Kuzmin, V. A. Rubakov and M. E. Shaposhnikov, Phys. Lett. B 155 (1985) 36.

22. P. H. Frampton, S. L. Glashow and T. Yanagida, Phys. Lett. B548 (2002) 119 [arXiv:hep-ph/0208157]; M. Raidal and A. Strumia, Phys. Lett. B553 (2003) 72 [arXiv:hep-ph/0210021].

23. S. Davidson and A. Ibarra, Phys. Lett. B535 (2002) 25 [arXiv:hep$\mathrm{ph} / 0202239]$.

24. W. Buchmuller, P. Di Bari and M. Plumacher, Phys. Lett. B 547 (2002) 128 [arXiv:hep-ph/0209301].

25. G. F. Giudice, A. Notari, M. Raidal, A. Riotto and A. Strumia, Nucl. Phys. B685 (2004) 89 [arXiv:hep-ph/0310123].

26. M. Flanz, E.A. Paschos and U. Sarkar, Phys. Lett. B345 (1995) 248; M. Flanz, E.A. Paschos, U. Sarkar and J. Weiss, Phys. Lett. B389 (1996) 693; A. Pilaftsis, Nucl. Phys. B504 (1997) 61; A. Pilaftsis, Phys. Rev. D56 (1997) 5431, [arXiv:hep-ph/9707235]; J. R. Ellis, M. Raidal and T. Yanagida, Phys. Lett. B 546 (2002) 228 [arXiv:hep-ph/0206300].

27. L. Covi, E. Roulet and F. Vissani, Phys. Lett. B 384 (1996) 169 [arXiv:hep$\mathrm{ph} / 9605319]$.

28. S. Davidson and R. Kitano, JHEP 0403 (2004) 020 [arXiv:hep-ph/0312007]; T. Hambye, Y. Lin, A. Notari, M. Papucci and A. Strumia, arXiv:hep$\mathrm{ph} / 0312203$.

29. M. Raidal, A. Strumia and K. Turzynski, arXiv:hep-ph/0408015.

30. E. Ma and U. Sarkar, Phys. Rev. Lett. 80 (1998) 5716 [arXiv:hepph/9802445]; T. Hambye, E. Ma, U. Sarkar, Nucl. Phys. B602 (2001) 23 [hep-ph/0011192].

31. G. D'Ambrosio, T. Hambye, A. Hektor, M. Raidal and A. Rossi, arXiv:hep$\mathrm{ph} / 0407312$.

32. Y. Grossman, T. Kashti, Y. Nir and E. Roulet, arXiv:hep-ph/0307081; G. D'Ambrosio, G. F. Giudice and M. Raidal, Phys. Lett. B575 (2003) 75 [arXiv:hep-ph/0308031].

33. M. A. Luty, Phys. Rev. D 45 (1992) 455; M. Plumacher, Z. Phys. C 74 (1997) 549 [arXiv:hep-ph/9604229]; M. Plumacher, Nucl. Phys. B 530 (1998) 207 [arXiv:hep-ph/9704231]; R. Barbieri, P. Creminelli, A. Strumia and N. Tetradis, Nucl. Phys. B 575 (2000) 61 [arXiv:hep-ph/9911315]; W. Buchmuller, P. Di Bari and M. Plumacher, Nucl. Phys. B 643 (2002) 367 [arXiv:hep-ph/0205349].

34. A. Pilaftsis and T. E. J. Underwood, Nucl. Phys. B 692 (2004) 303 [arXiv:hep-ph/0309342].

35. M. Y. Khlopov and A. D. Linde, Phys. Lett. B 138 (1984) 265; J. R. Ellis, D. V. Nanopoulos and S. Sarkar, Nucl. Phys. B259 (1985) 175; J. R. Ellis, D. V. Nanopoulos, K. A. Olive and S. J. Rey, Astropart. Phys. 4 (1996) 371; M. Kawasaki and T. Moroi, Prog. Theor. Phys. 93 (1995) 879. 